\title{
Robust mine schedule optimisation
}

\author{
M Whittier MIRARCO Mining Innovation, Canada
}

R Hauta MIRARCO Mining Innovation, Canada

L Fava MIRARCO Mining Innovation, Canada

\begin{abstract}
The identification of high-value, practical mine plans is a complex and challenging process. Mineral prices are a crucial factor to the value of a project and, due to the inherent volatility of the commodities market, also the most uncertain. Despite this reality, conventional planning processes don't sufficiently account for the financial uncertainty associated with mining assets. As a consequence, life-of-mine schedules must often be extensively revisited as the fiscal climate changes. The inherent financial uncertainty of mining projects presents a substantial risk to stakeholders and without proper mitigation can easily reduce the perceived and real value of a mining asset.

The risks associated with uncertainty can be mitigated through the creation of robust mine schedules. In this paper, two distinct approaches to creating robust, optimised long-term underground mine schedules will be presented. The Genetic Optimizer for Stochastic Problems (GOSP) method incorporates price distributions, rather than fixed projections, into the optimisation process. The Horizon method incorporates the concept of 'management flexibility' into the process of schedule optimisation. As will be demonstrated by a case study, these methods, when integrated with the Schedule Optimization Tool (SOT), produce robust, high net present value schedules for underground mining operations.
\end{abstract}

Keywords: robust mine planning, schedule optimisation, financial uncertainty, genetic algorithm

\section{Introduction}

Even with investors and economists having developed a variety of commodity price-forecasting methodologies, current long-term and mid-term price-predicting practices remain unreliable. The range of current commodity price-forecasting approaches includes qualitative methods (judgemental forecasting), cost-reserve based methods, trend extrapolation methods, time series methods, causal models and even the use of futures markets. The selection of the correct price-forecasting technique is determined according to the time period under consideration (short, medium or long-term) since prices and trends relating to each of these time periods are driven by very different factors (Labys 2006). Regardless of the vast body of work pertaining to these concepts, as expressed by Van Rensburg (1978) and maintained by the findings of others (Dooley \& Lenihan 2005; Bowman \& Husain 2004), forecasting remains an art, rather than a science.

Without reliable price predictions, strategic mine plans are subject to a large amount of financial risk resulting from uncertainty. Despite these challenges, modern computing power gives planners the ability to mitigate the risks of price uncertainty without sacrificing high-value schedules. In this paper, two methods of robustly optimising underground mine schedules will be presented: the Genetic Optimizer for Stochastic Problems (GOSP) method and the Horizon method. Their applications as scheduling tools will be presented as a case study, which will demonstrate how financial risk can be reduced through robust schedule optimisation.

\subsection{Mine planning software}

Most scheduling software approaches the strategic planning process by dividing and categorising all major tasks associated with the development and production of a mining project. Usually referred to as activities, these tasks represent the major distinct actions to be carried out as a part of a mining operation. Activities 
usually include production drilling, ore mucking, lateral development, vertical development and backfilling. A duration or rate is assigned to each activity along with relevant production and classification attributes. Examples of production attributes are grade, tonnage, length, and fill tonnes. Classification attributes are important descriptors of the tasks such as the mine area, level, or zone.

To construct a schedule, activities are first related to one another by means of precedence constraints, commonly referred to as dependency links. A dependency link is used to specify that a 'predecessor' activity must be completed before a 'successor' activity may start. For example, an access development must be completed before the mining of the associated stope can begin. Using the activity durations and the dependency links, exact 'start' and 'finish' times are calculated for every task. In order to generate feasible schedules, production and custom attribute constraints are included in the exact date calculations.

\subsection{Schedule optimisation}

There are several commercially available mine scheduling software packages that are structured in this manner. Additionally, schedule optimisation programs exist that complement these software packages. The vast majority of schedule optimisation programs with the primary goal of maximising a project's net present value (NPV) are available for open pit applications only.

\subsubsection{Underground schedule optimisation}

Classical optimisation methods attempt to analytically identify a provably optimal solution. However, the computational complexity of the underground scheduling problem makes this approach infeasible for the large search space that mining projects present. Hence, classical optimisation approaches necessitate aggregation of activities and the use of large scheduling periods, such as annual. For this work, the activities remain at the desired level of detail and they are assigned precise start times.

Schedule optimisation seeks to maximise or minimise relevant project valuation or operational parameters while adhering to project constraints. Typically, the objective is to maximise the NPV of an asset. In order to be considered feasible, the schedules generated must adhere to all precedence constraints, production and classification attribute capacities, and operational resource constraints.

Evolutionary algorithms, employing principles inspired by biological adaptation, have been particularly useful for finding satisfactory solutions to intractable problems. A particular subset of evolutionary learning algorithms, genetic algorithms, achieve this through the use of operators which mimic primary concepts of natural selection. These genetic operators, selection, crossover, mutation and elitism, have been shown to increase the effectiveness of the search for high-quality schedules. Despite not generating provably optimal solutions, they provide significant improvements over manually identified solutions or exact solutions to aggregated versions of the scheduling problem.

\subsubsection{The Schedule Optimization Tool}

The Schedule Optimization Tool (SOT) (Maybee et al. 2010), was utilised for this case study. SOT takes as input a set of activities and corresponding dependency links along with other project constraints related to production and classification attributes. SOT is commercial software that utilises an evolutionary algorithm and heuristics to efficiently generate high-value schedules. Heuristics are simple logical rules that can very quickly generate favourable initial schedules. An iterative search process generates a population of feasible schedules and calculates their NPVs. The best schedules from the population are brought forward to the next iteration, and the remainder of the population is modified with various genetic operators. This iterative process continues until the learning process converges. Learning convergence is defined as multiple iterations occurring with no significant improvement to the NPV of the best schedule present in the population. An overview of this optimisation process is presented in Figure 1. 


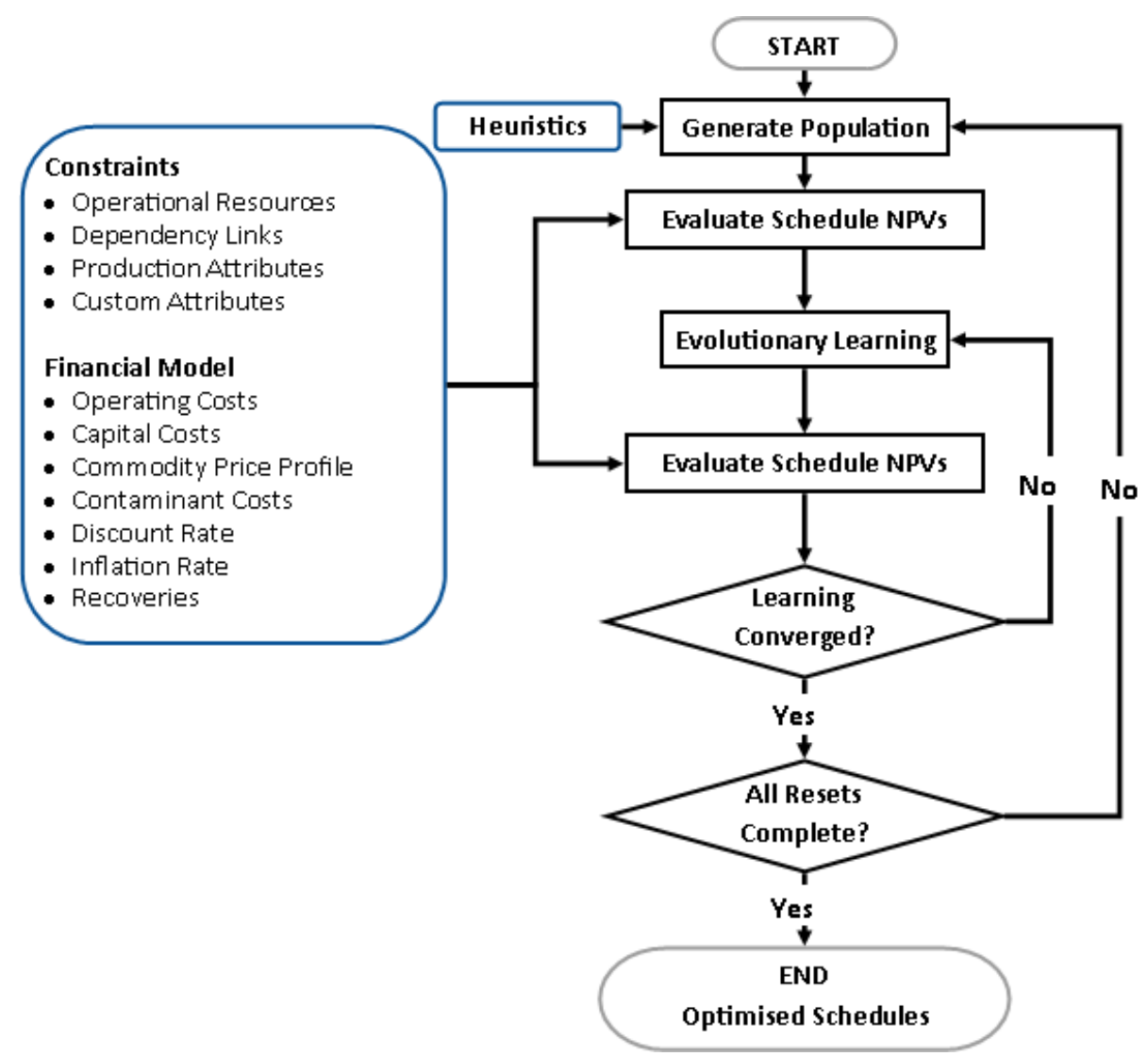

Figure 1 Process applied by SOT for optimising underground mine schedules

\subsection{Commodity price uncertainty}

Commodity prices are the single largest inherent uncertainty related to mining projects. A sensitivity analysis on any mining project will almost always indicate that the value of a mining asset is most sensitive to changes in commodity prices. Accordingly, commodity price uncertainty and price volatility presents a major source of financial risk to project schedules. In reference to strategic planning, price uncertainty is rarely addressed and most schedule optimisation is conducted using constant or fixed ascending prices. At best, planners will create multiple mine plans, corresponding to different price scenarios, as a means of preparing for different price outcomes. Even so, it is highly improbable that any schedule optimised with fixed price assumptions could still be considered optimised during project execution. Quite simply, by not incorporating price uncertainty into the strategic planning process, project value is being lost.

\subsection{Robust schedule optimisation}

In order to attain full value from a mining asset, strategic plans should be produced, assessed and optimised based on the fact that mine plans can be subject to a broad range of commodity price behaviours over the life of a project. By incorporating price uncertainty directly into the optimisation process, high-quality schedules that also mitigate risks associated with price uncertainty can be generated. A robustly optimised schedule is defined here as a schedule that, compared to schedules optimised more conventionally, is most likely to deliver high value under a variety of plausible price outcomes. 


\subsubsection{Genetic Optimizer for Stochastic Problems}

The GOSP approach to robustly optimising schedules incorporates multiple product price realisations into the optimisation process (Saavedra-Rosas 2009). Integrated with the SOT process of Figure 1, GOSP enhances the assessment of schedule value by considering many alternative product price realisations at each iteration of the evolutionary process. The work reported here enhanced GOSP with an alternative objective function and an additional measure of schedule robustness, below-target semivariance, as given by Equation 1 .

$$
S V_{t}=\frac{1}{n}\left(\sum_{b=1}^{n}\left(t-r_{b}\right)^{2}\right)
$$

where:

$t \quad=$ target NPV, equal to the median NPV of the population.

$n \quad=$ number of price scenarios where schedule NPV was less than $t$.

$r_{b} \quad=$ a below-target NPV of the schedule.

Semivariance, an essential tool in modern portfolio theory, is one of two most commonly used downside risk measures in the management of risky investments (Nawrocki 1999). The best schedules in the GOSP approach are defined as schedules that are non-dominated in terms of NPV and below-target semivariance when evaluated with numerous commodity price scenarios.

The GOSP-SOT process begins with the generation of a population of schedules. The NPV of each schedule in the population is evaluated with numerous commodity price scenarios; these are then aggregated into an average value to facilitate identifying the best schedules while accounting for the effects of different possible price outcomes. Multiple iterations of schedule improvement are carried out until the process begins to converge, at which time the number of price scenarios is increased. The overall process terminates when the population has converged, meaning that the addition of new scenarios doesn't significantly change the population of schedules - they have become robust. This robust optimisation process is depicted in Figure 2 .

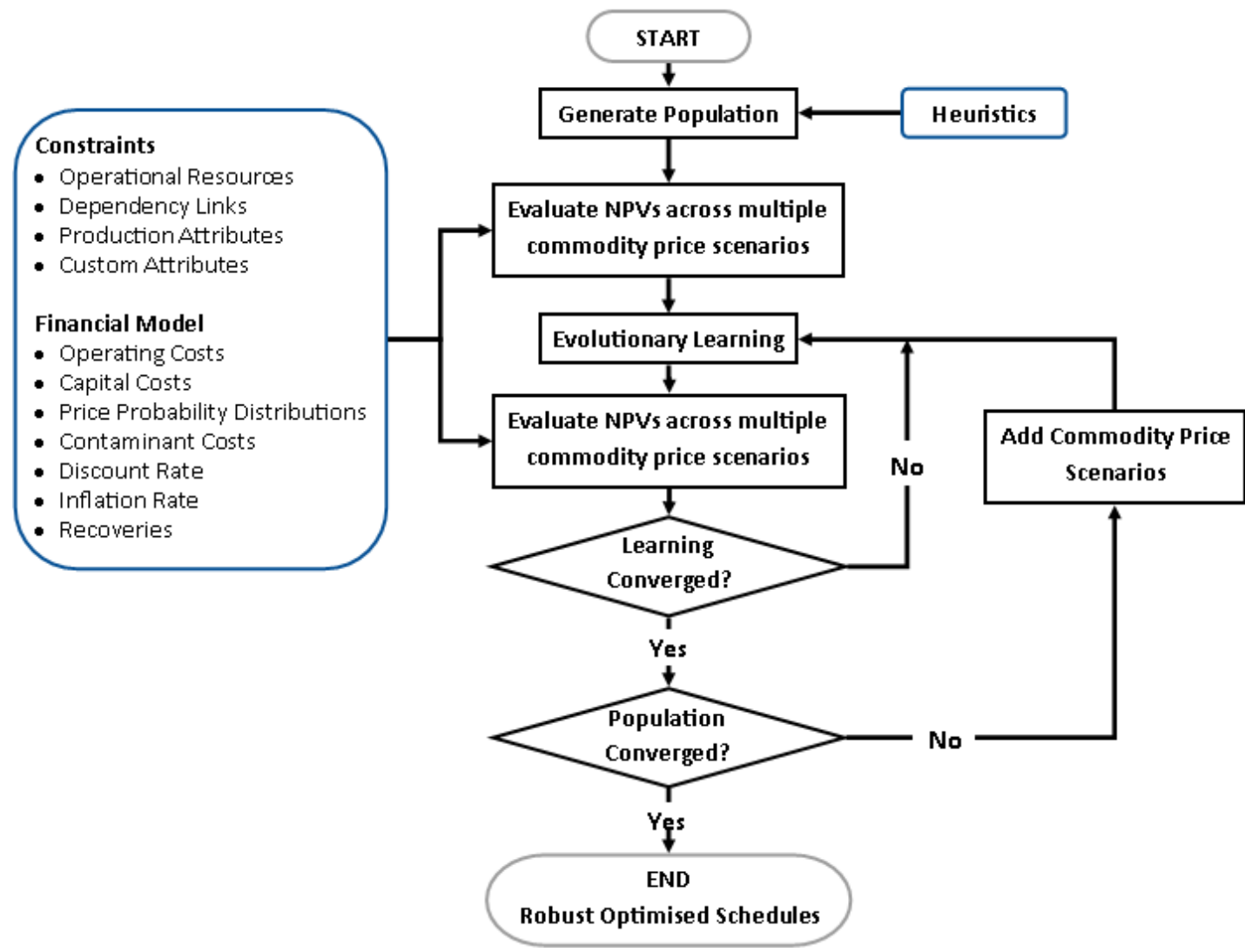

Figure 2 Process employed by the GOSP-SOT method for generating robustly optimised underground mine schedules 


\subsubsection{The Horizon method}

The Horizon method is another approach to robust optimisation that incorporates commodity price uncertainty, but also concepts of managerial flexibility and real options analysis (Maybee 2010). Managerial flexibility refers to the ability of decision-makers to adapt plans in response to unexpected outcomes in order to influence the value of real (non-financial) assets. In general, this can include adaptations in response to anything from market conditions to operational delays. Real options, as defined by Triantis (2000), "are opportunities to delay and adjust investment and operating decisions over time in response to the resolution of uncertainty". When decision-makers take advantage of real options, risk can be mitigated and opportunities even provided to exploit the uncertainty of future outcomes. "More broadly, the real options approach is a way of thinking that helps managers formulate their strategic options" (Amram \& Kulatilaka 2000). Likewise, real options valuation is an alternative asset valuation approach which, unlike the conventional discounted cash flow (DCF) method, considers different strategic options and accounts for the value that managerial flexibility contributes to an asset (Jaimungal \& Lawryshyn 2011). Rather than assuming that a predetermined plan will be followed regardless of how events unfold, real option methods incorporate both the risk associated with uncertainty and active decision-making into the valuation process (Luehrman 1998).

The Horizon method borrows from these concepts and implements a method where the early phases of the project are differentiated from the later time periods of the project with regard to their level of uncertainty. A horizon (or horizon date) is selected to distinguish the date where the known time period transitions into an uncertain future.

The Horizon method has been integrated with SOT in the context of commodity price uncertainty. For the period before the horizon, there is a confidence that an optimised solution can be generated based on price projections. Typically, for later periods in the mine life, commodity price projections have greater uncertainty and higher associated risk. The Horizon method endeavours to generate a schedule with an early phase that can seamlessly transition into whatever schedule strategy needs to be pursued after the horizon date as a result of the prices that are realised.

First, the SOT-Horizon method generates an optimised schedule, and locks the activity dates prior to the horizon date. The portion of the schedule after the horizon is then re-optimised using SOT, with a commodity price scenario derived from a probability distribution. To evaluate the sensitivity and robustness of the pre-horizon schedule to price uncertainty, many post-horizon schedules are optimised according to different price scenarios. A conceptual representation of the products of the SOT-Horizon method can be found in Figure 3 .

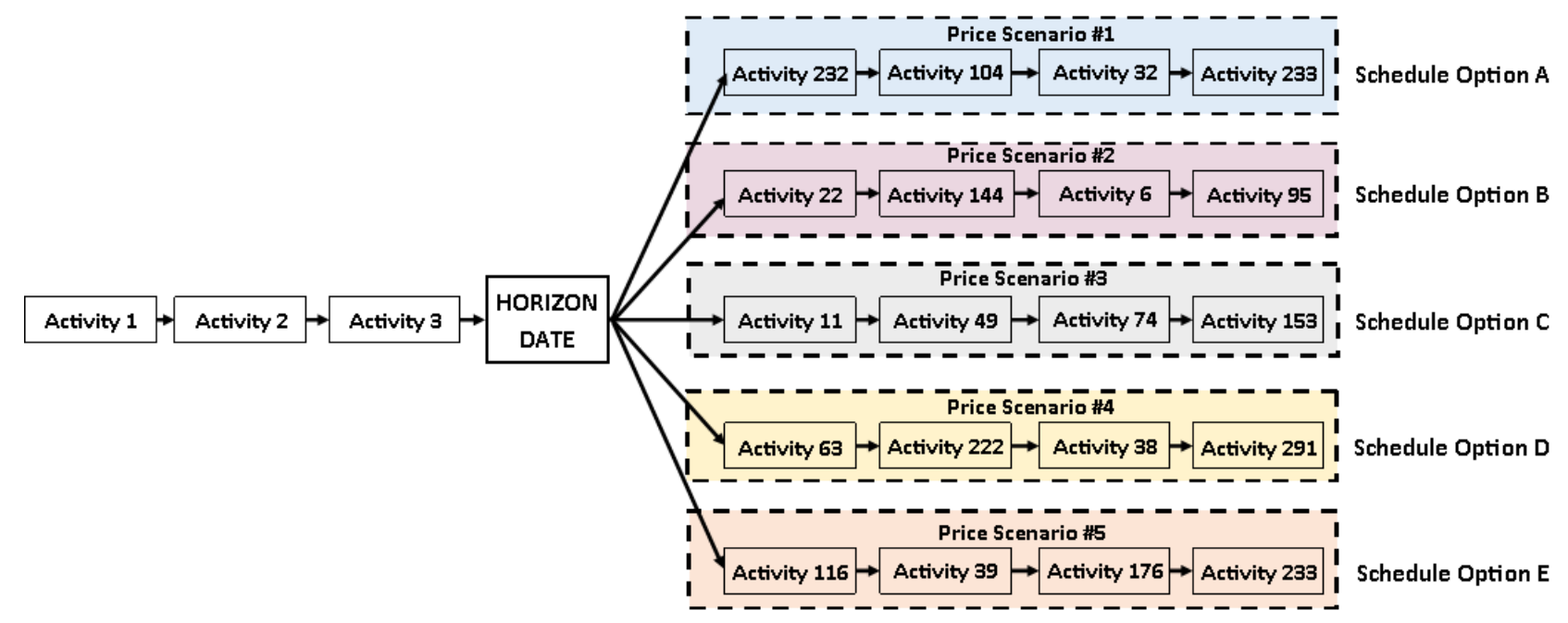

Figure 3 Conceptual representation of the SOT-Horizon method's structure of optimised schedule options 


\section{Case study}

\section{$2.1 \quad$ Data}

The dataset used to conduct the case study consists of an underground mining project with a steeply dipping, narrow vein orebody. It is a poly-metallic deposit comprising of gold with secondary products silver and copper. The mine has both shaft and ramp access with a shaft hoisting capacity of 1,700,000 t per year. Several mining methods are employed including longhole stoping, conventional cut-and-fill, mechanised drift-and-fill and alimak stoping. The plant recovery is $94 \%$ and the gold cut-off grade defined at $1.7 \mathrm{~g} / \mathrm{t}$. The estimated mine life is over 30 years.

\subsection{Methodology}

\subsubsection{Modelling commodity price uncertainty}

A key aspect to incorporating commodity price uncertainty into the process of producing robust mine schedules is the manner in which the price uncertainty is modelled. To assess robustness and uncertainty while minimising bias, probability distributions were used to generate price scenarios. Using a commodity price probability distribution defined from historical data, prices were randomly generated from the probability distribution for every year of the mine life. Metal price scenarios for both the robust optimisation process and analysis of the results were produced in this manner.

Triangular probability distributions for gold, silver and copper were defined from monthly average historical price data. The probability distribution representing possible price outcomes for the projects products are plotted in Figures 4, 5 and 6. Historic data in USD from 1972 to 2016, taken from The World Bank (2017) Pink Sheets was used. Metal prices prior to 1972 were omitted since the price of gold was only freed by the United States government when the gold standard was rescinded in 1971.

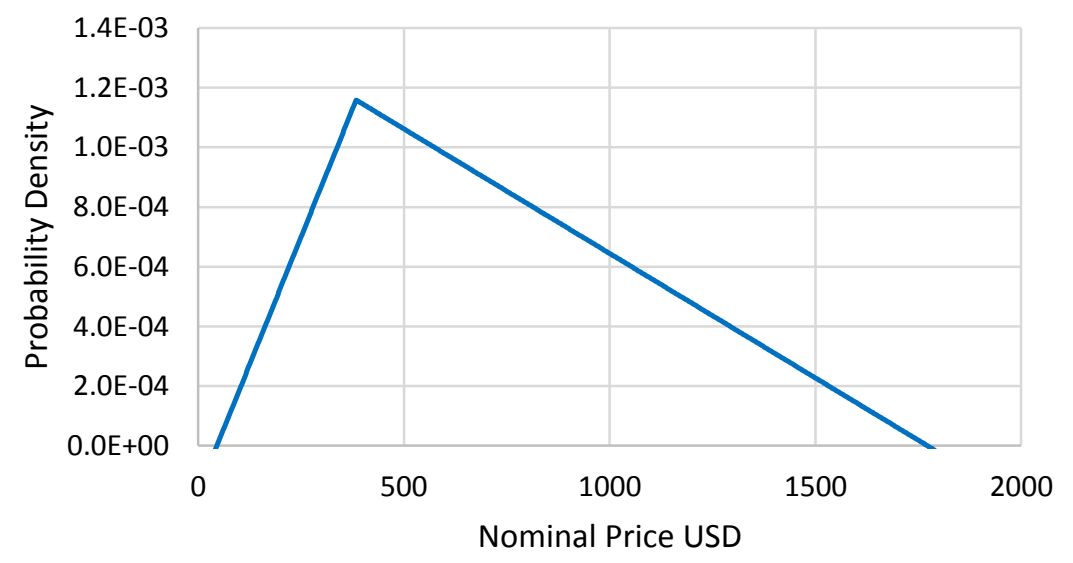

Figure 4 Gold price probability distribution used to generate different gold price scenarios 


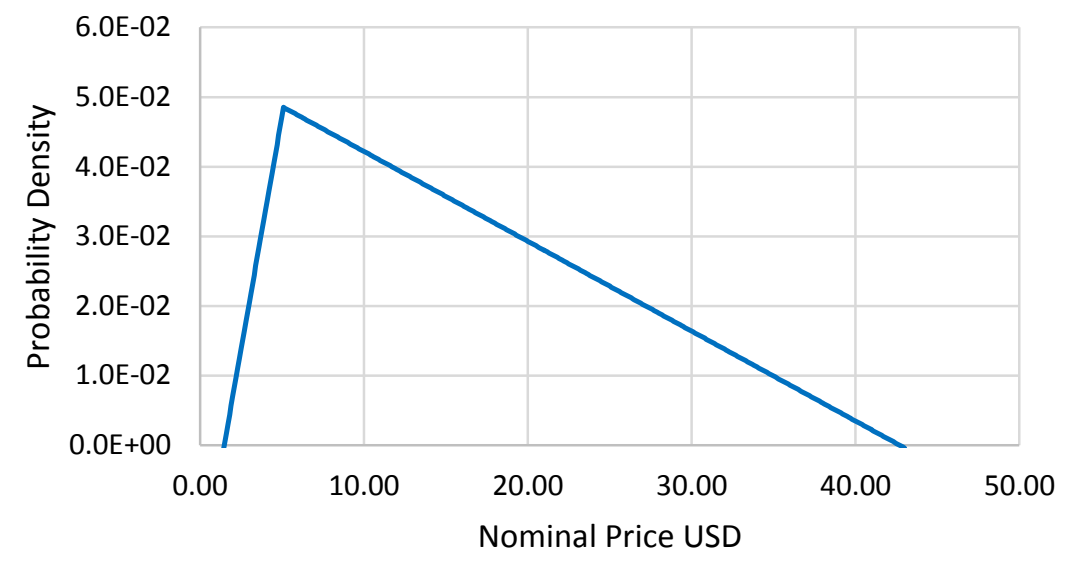

Figure 5 Silver price probability distribution used to generate different gold price scenarios

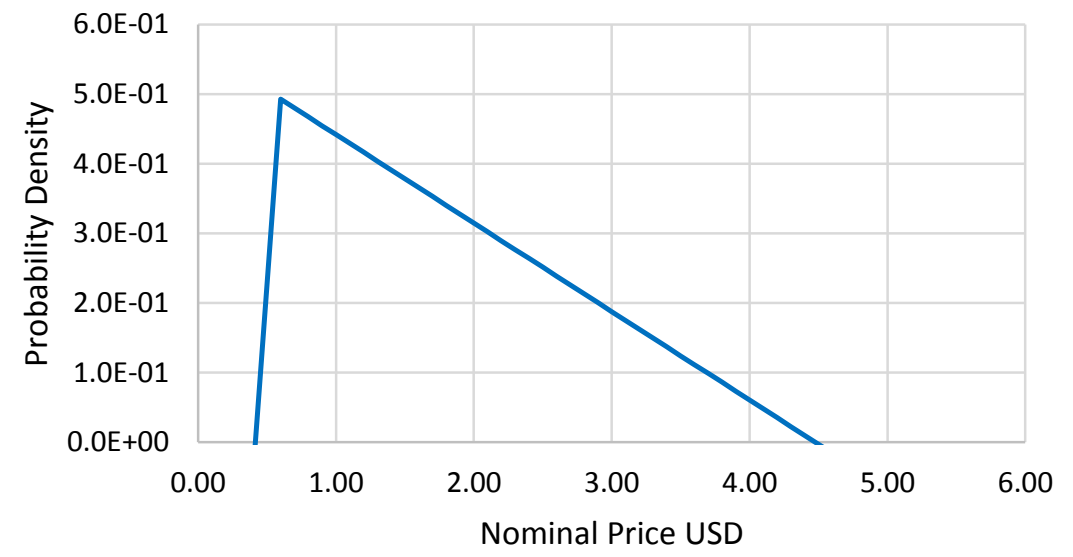

Figure 6 Copper price probability distribution used to generate different gold price scenarios

\subsubsection{Underground mine schedule optimisation}

One standard SOT-optimised schedule was generated for the purpose of comparing to SOT-GOSP schedules. Two additional SOT-optimised schedules were produced for valuation using the Horizon method.

Standard underground mine schedule optimisations were conducted using SOT as described in Section 1.2.2. Constant metal prices were used for all the standard SOT optimisations. Metal prices used are those forecasted by the mining company when conducting their own project valuations. A discount rate of $7 \%$ was used for NPV calculations, as considered appropriate for the region.

The two schedules produced for valuation using the Horizon method were generated using different heuristics in order to produce schedules reflecting different planning strategies.

\subsubsection{The GOSP-SOT method}

The GOSP-SOT method was carried out in order to robustly optimise a schedule to achieve a best-expected NPV instead of merely a best NPV. Except for the metal prices, the project was configured in the same manner as the standard schedule optimisations outlined in Section 2.2.2. Price probability distributions were defined for gold, silver and copper as outlined in Section 2.2.1. The criterion for population convergence (refer to Figure 2) requires that $5 \%$ or more of the schedule population remains unchanged. Every full iteration, 20 commodity price scenarios were added. 
The robustly optimised schedule (GOSP-SOT schedule) and standard optimised schedule (SOT schedule) were then evaluated with 100 different price scenarios and compared in terms of their downside risk and overall schedule value.

\subsubsection{The SOT-Horizon method}

A practical scenario where the mining company has a set price contract for the first nine years of the project for all three of their metal products was used. The SOT-Horizon method was then carried out using this scenario to assess and compare the robustness of two separate schedules when considering the schedules themselves as well as the real options each schedule allows for in the future.

The two schedules were produced and optimised using different heuristics in SOT in order to produce two unique schedules that apply different planning strategies. Using the Horizon method, the dates on both schedules were locked for the first nine years while prices were guaranteed by company contracts. The schedules after the contract conclusion date were then subject to ten different price scenarios and optimised according to those price scenarios. Ten different schedules, considered possible options available to pursue after year nine of the initial SOT schedule, were produced for each of the two schedules. The two initial schedules and corresponding 10 best schedule options were then used to identify which of the two schedules is indeed more favourable in terms of its value and downside risk.

\subsubsection{Quantifying risk}

The source of risk that was assessed in this case study consisted of the financial risk associated with commodity price uncertainty and its negative impacts on the overall value of the mining project. The schedule outcomes that fell below a target project value were the focus of the risk analysis. The 'risk' of positive financial outcomes was not included in the risk assessment, as positive outcomes are generally not considered a consequence of risk that require management or mitigation.

Schedules were valued in terms of NPV, calculated using the conventional DCF method. Schedules with highly variable NPV results below the target value, when subject to a variety of possible price scenarios, were classified as schedules that presented higher risk. The downside risk of each schedule was quantified in terms of the below-target semivariance calculated using Equation 1 defined in Section 1.4.1. The target value utilised in the case study consisted of the median NPV of all of the schedule values resultant from their evaluation using various price scenarios.

\section{$2.3 \quad$ Results}

\subsubsection{GOSP-SOT}

The best 27 schedules out of the 356 produced by a GOSP-SOT optimisation are depicted in Figure 7 in terms of their average NPV and below-target semivariance. The results of a standard SOT-optimised schedule is also shown for comparison. Each schedule was evaluated using the same 100 price scenarios. 


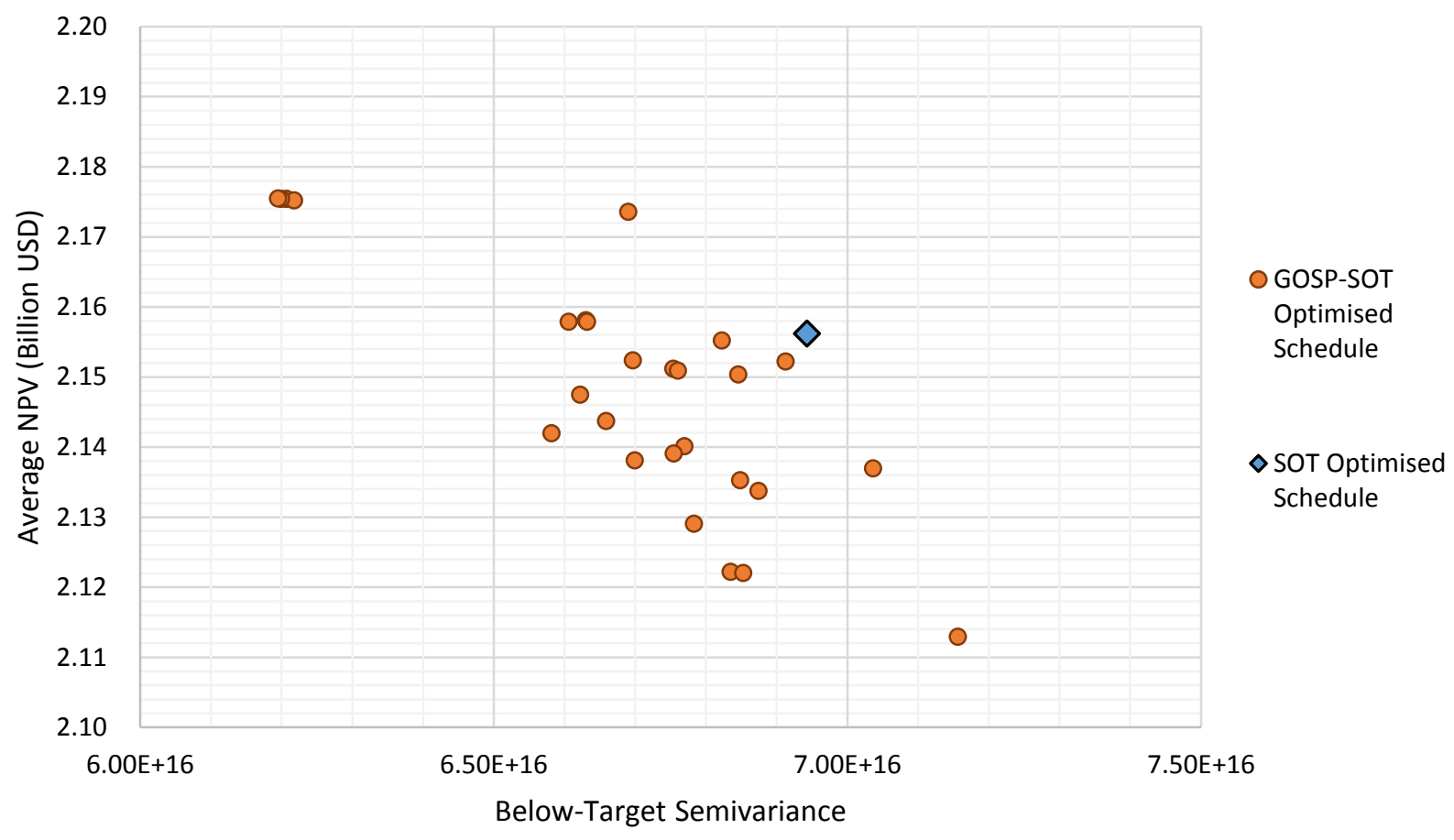

Figure 7 SOT and GOSP-SOT schedules evaluated with 100 different price scenarios

Of the 27 best GOSP-SOT schedules, eight were comparable if not superior to the SOT-optimised schedules based on both their NPV and below-target semivariance.

The below-target semivariance for the SOT-optimised schedule and best GOSP-SOT-optimised schedule is summarised in Table 1. The schedule produced, using the GOSP method, exhibits a lower NPV dispersion below the target than the standard SOT-optimised schedule. The $11 \%$ reduction in downside variability suggests that the risk of negative financial outcomes is lower for the GOSP-SOT produced schedule when subject to volatile price behaviour.

Table 1 GOSP-SOT and SOT-optimised schedules calculated below-target semivariance for 100 different price scenarios

\begin{tabular}{ll}
\hline Schedule & Below-target semivariance \\
\hline Standard SOT optimisation & $6.943 \mathrm{E}+16$ \\
Best GOSP-SOT optimisation & $6.195 \mathrm{E}+16$ \\
\hline
\end{tabular}

The distribution of schedule NPVs for both the SOT-optimised schedule and GOSP-SOT-optimised schedule when subject to the various price scenarios are depicted in Figure 8. The whiskers on the boxplot depict the maximum and minimum NPV resulting from the various scenarios. The box plot shows that in addition to less downside risk, the best GOSP-SOT schedule also has a higher median NPV than the standard SOT schedule.

Considering these parameters together, it is clear that a schedule that is both robust and economically superior can be produced by applying the GOSP method. 


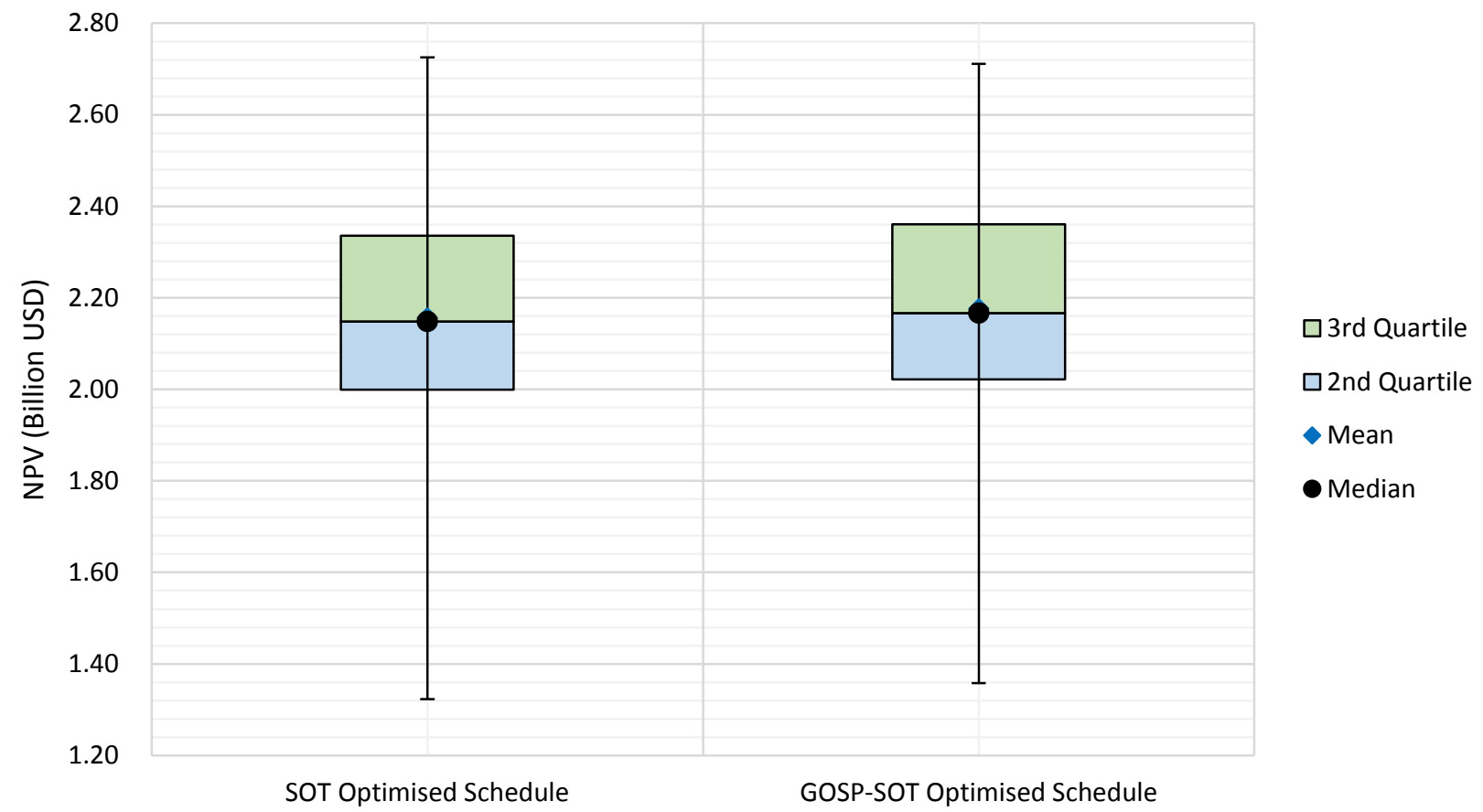

Figure 8 NPV boxplot results for the SOT schedule and best GOSP-SOT schedule evaluated using 100 price scenarios

\subsubsection{The SOT-Horizon method}

The schedule results for the two SOT-optimised schedules that would conventionally be considered in order to select a schedule for implementation are summarised in Table 2. The NPV was calculated using the conventional DCF method with company forecasted constant metal prices. The below-target semivariance was calculated using 1,000 price scenarios.

Table 2 NPV using company forecasted prices and below-target semivariance when evaluated with 1,000 price scenarios

\begin{tabular}{lll}
\hline Schedule & NPV & Below-target semivariance \\
\hline Schedule A & USD 955,069,877 & $3.691 \mathrm{E}+16$ \\
Schedule B & USD 945,020,533 & $3.803 \mathrm{E}+16$ \\
\hline
\end{tabular}

When considering these conventional parameters, the schedules are comparable with regard to their economic value and downside risk. When selecting one for implementation, Schedule A would be considered advantageous as it has a higher NPV and lower below-target semivariance. However, when the best ten options available for each of the two schedules were considered using the Horizon method, Schedule B was determined to be superior. The first indication of this is when considering and comparing the robustness of the best 10 schedule options for each SOT-optimised schedule. The below-target semivariance for each of the ten schedule options for both SOT schedules were calculated and are plotted in Figure 9. 


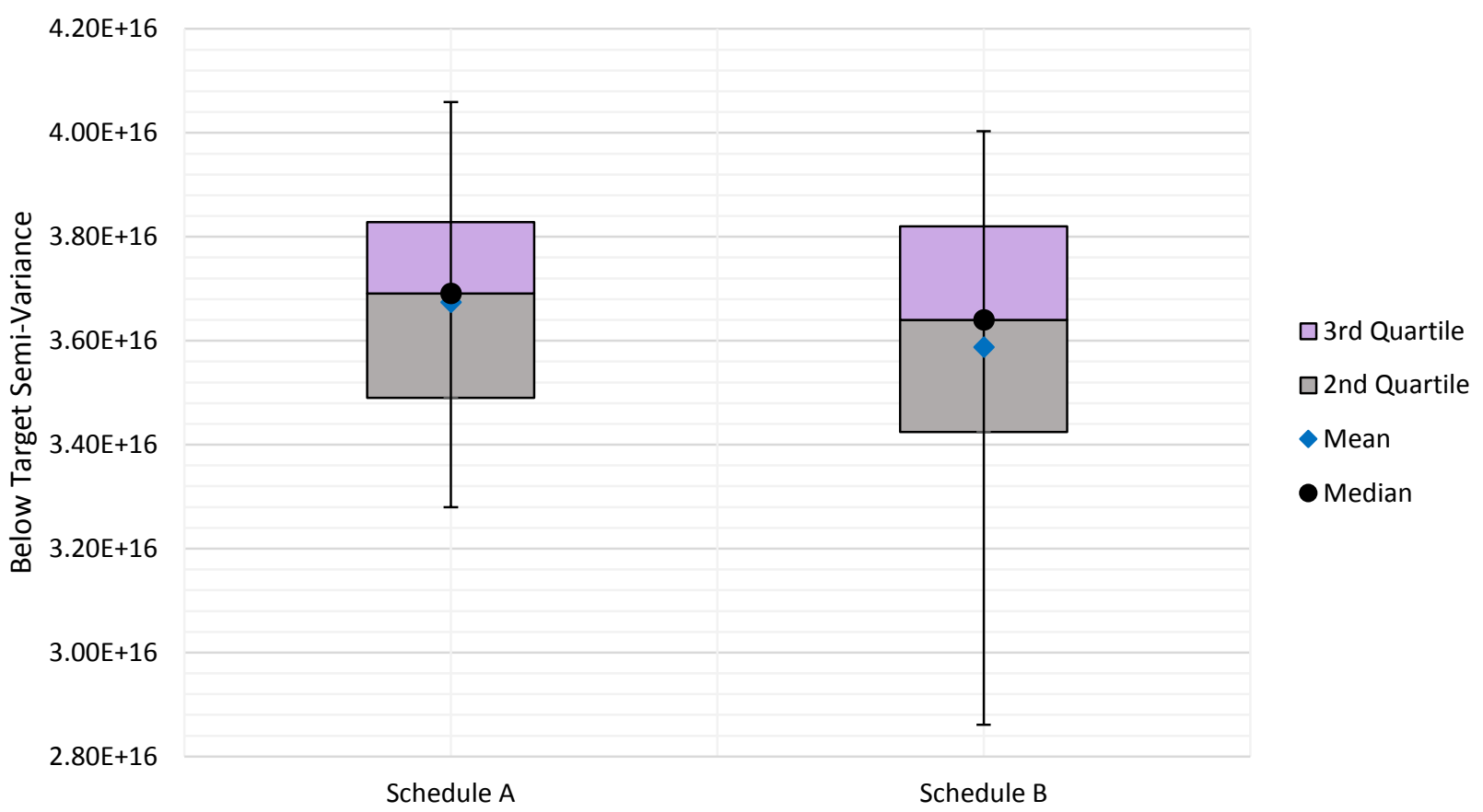

Figure 9 Below-target semivariance for the 10 schedule options for Schedules 1 and 2

The below-target semivariance was found to be lower for the Schedule B options, most notably with regard to the median schedule semivariance of all the schedule options. In fact, all the bounds depicted in the plot demonstrate a lower below-target semivariance for the Schedule B options. The minimum and $25^{\text {th }}$ percentile bounds in particular demonstrate a drastically lowered semivariance with the Schedule B options. This result indicates that there is a greater likelihood of achieving a lower semivariance by pursuing Schedule B over Schedule A. Furthermore, it quantifies that when considering the ten best options for both schedules, the risk is lower for Schedule B than for Schedule A.

The median NPV for all Schedule B options were found to be larger than all of the median values for the Schedule A options. Likewise, the $75^{\text {th }}$ percentile for most of the Schedule B options were found to have higher NPVs than the Schedule A options. All of this illustrates that if Schedule B were altered in order to pursue one of its best options, then the NPV would likely be higher than any of the best Schedule A options that could be pursued. These valuation results from 1,000 price scenarios for Schedule A and Schedule B, along with their best 10 schedule options, are depicted in Figures 10 and 11 . The target value depicted in the plot was the same target value used to calculate the below-target semivariance. 


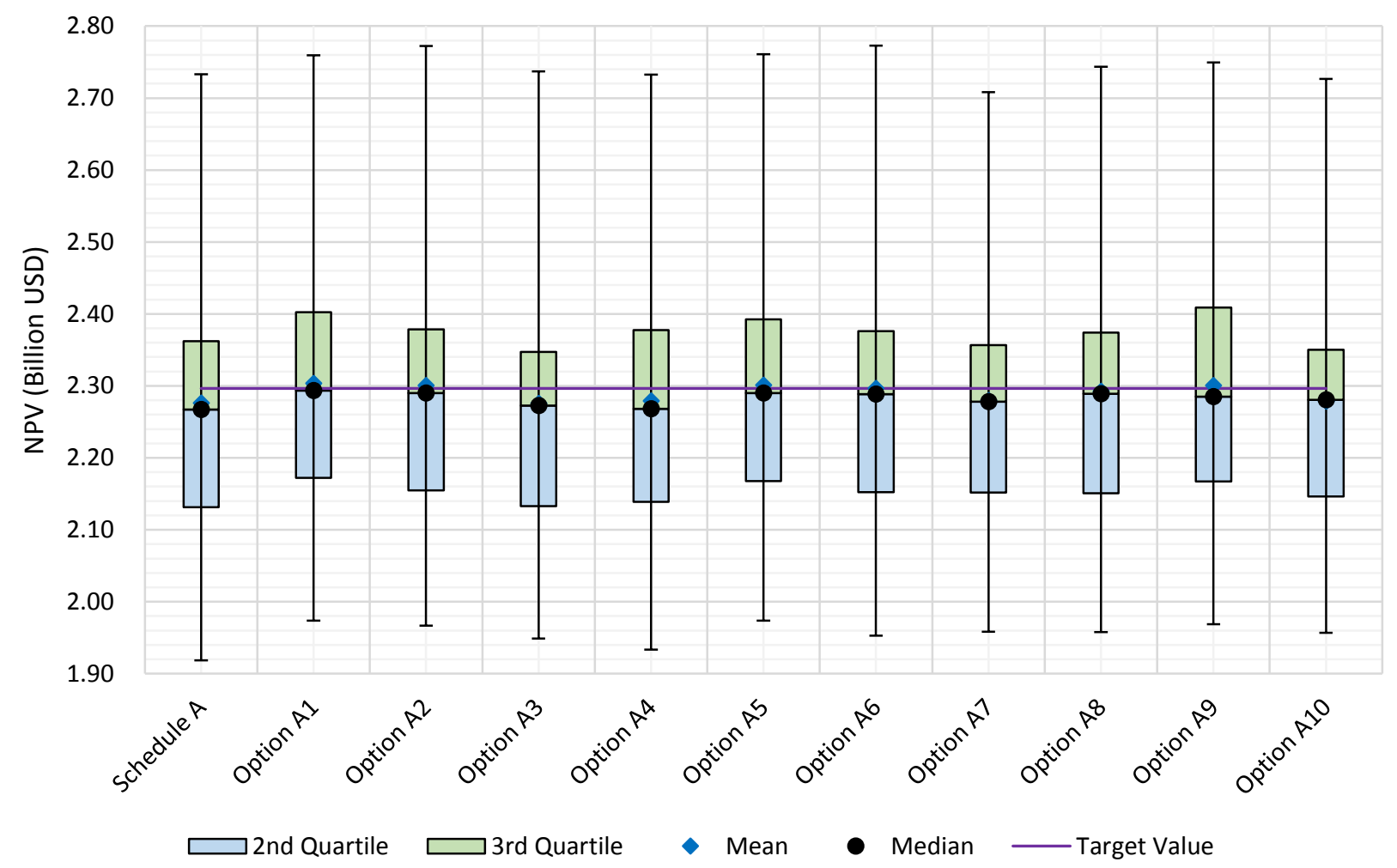

Figure 10 NPV boxplot for Schedule A and 10 best schedule options that can be pursued after year nine

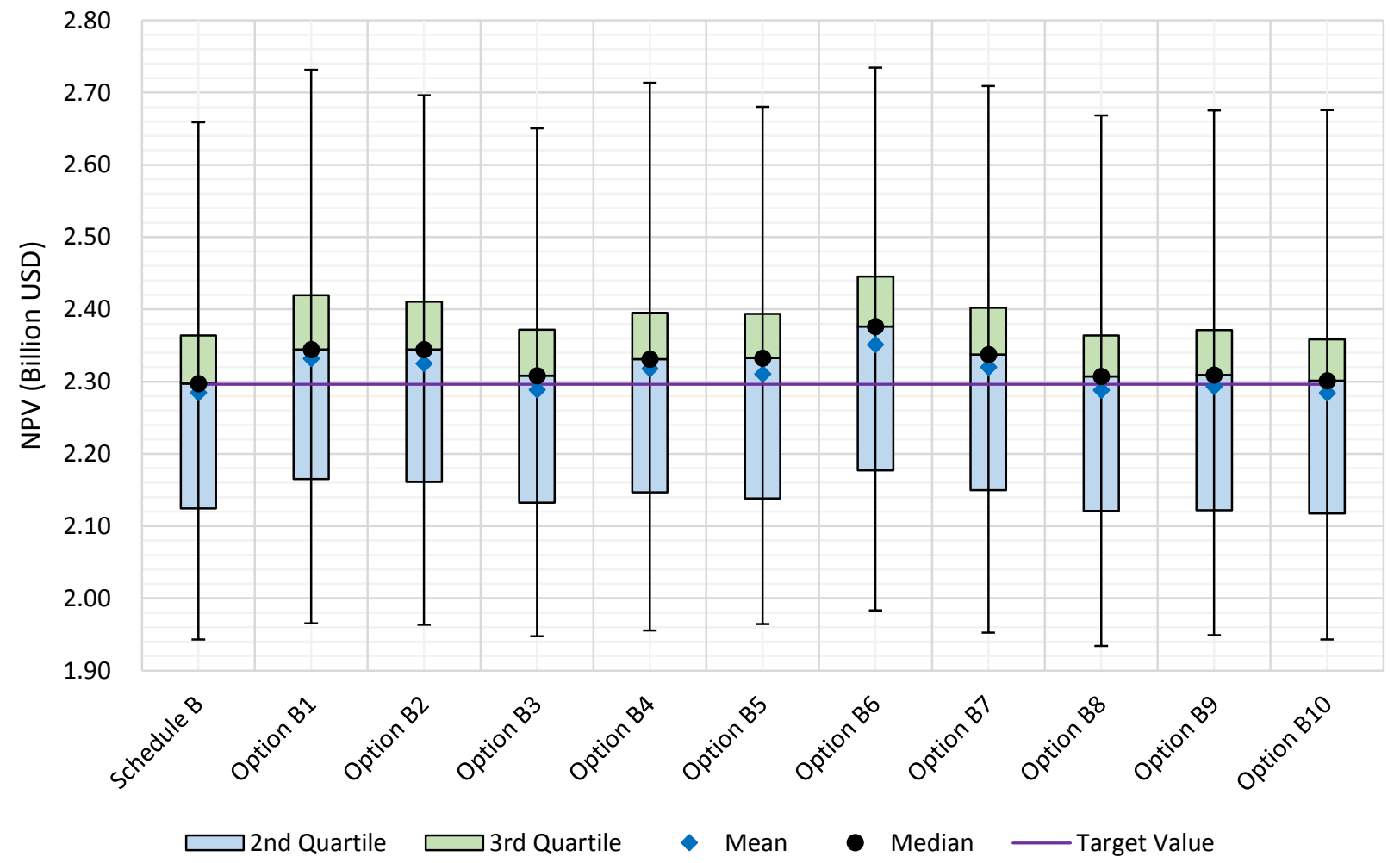

Figure 11 NPV boxplot for Schedule B and 10 best schedule options that can be pursued after year nine 
From both these measures, it is apparent that Schedule B is the superior schedule when realistic and likely changes to initial schedules are considered. This conclusion contradicts those drawn using conventional valuation methods. This is significant as it demonstrates how consideration of real schedule options using the Horizon method during the valuation and risk assessment process can increase potential value that would have otherwise been lost.

\section{Conclusion}

Often an intended strategic plan cannot be implemented exactly as envisioned, and a major alteration to the schedule is made at some point during the life of the project. By applying the Horizon method to consider the various schedule options available to pursue for a candidate schedule, a better picture of the candidate's plan value and associated risk can be determined. As the results of the case study demonstrate, considering a schedule's real options can alter the choice of strategic plan by identifying a superior solution. Likewise, it was illustrated that without considering the real options of a schedule during the valuation and risk assessment process, potential project values may not be realised.

Similarly, by considering a variety of price scenarios within the schedule optimisation process, it was established that the risk resulting from commodity price uncertainty could be mitigated. Through the application of the GOSP method, schedules with a reduced below-target semivariance were generated. Moreover, the schedules produced in this manner exhibit superior NPV outcomes, when subject to various price scenarios, compared to those optimised from fixed price projections.

\section{Acknowledgements}

The Horizon method was established by Bryan Maybee, now with Curtin University, and the GOSP method by José Saavedra-Rosas, now with Coalesce Group, during their prior work at MIRARCO and as part of their PhD theses. The authors thank Darren Janeczek, Scott McGarvey, and Chris Mangiardi of MIRARCO for their valuable assistance with this work. The significant contributions to the SOT+ research project by Newmont, Vale Canada, Agnico Eagle, Deswik, Datamine, and the Ultra-Deep Mining Network (UDMN) at the Centre for Excellence in Mining Innovation (CEMI) are gratefully acknowledged.

\section{References}

Amram, M \& Kulatilaka, N 2000, 'Stategy and shareholder value creation: the real options frontier', Journal of Applied Corporate Finance, vol. 13 , no. 2, pp. 18-28.

Bowman, C \& Husain, AM 2004, Forecasting Commodity Prices: Futures Versus Judgement, International Monetary Fund, Washington, DC, viewed 16 May 2017, https://www.imf.org/external/pubs/ft/wp/2004/wp0441.pdf

Dooley, G \& Lenihan, H 2005, 'An assessment of time series methods in metal price forecasting', Resources Policy, vol. 30, no. 3, pp. 208-217.

Jaimungal, J \& Lawryshyn, Y 2011, 'Incorporating managerial information into valuation of early stage investments', Proceedings of the 15th Annual International Conference on Real Options: Theory Meets Practice, Real Options Group, Turku.

Labys, W 2006, Modelling and Forecasting Primary Commodity Prices, Ashgate Publishing Ltd., Farnham.

Luehrman, T 1998, 'Strategy as a portfolio of real options', Harvard Business Review, vol. 76, no. 5, pp. 89-99.

Maybee, B 2010, A Risk-based Evaluation Methodology for Underground Mine Planning, PhD thesis, Natural Resources Engineering, Laurentian University, Sudbury.

Maybee, B, Fava, L, Dunn, PG, Wilson, S \& Fitzgerald, J 2010, 'Towards optimum value in underground mine scheduling', CIM Journal, vol. 1, no. 3, pp. 176-182.

Nawrocki, D 1999, 'A brief history of downside risk measures', The Journal of Investing, vol. 8, no. 3, pp. 9-25.

Saavedra-Rosas, J 2009, A Genetic Optimizer for Stochastic Problems with Applications to Orebody Uncertainty in Mine Planning, PhD thesis, Natural Resources Engineering, Laurentian University, Sudbury.

The World Bank 2017, Commodity Price Data (The Pink Sheets), The World Bank, Washington, DC, viewed 6 February 2017, http://www.worldbank.org/en/research/commodity-markets\#1

Triantis, A 2000, 'Real options and corporate risk management', Journal of Applied Corporate Finance, vol. 13, no. 2, pp. 64-73.

Van Rensburg, WCJ 1978, The Economics of the World's Mineral Industries, McGraw-Hill, Johannesburg. 
